$<80 \%$ (3.29 vs. 1.21) per hour decreased. No heart block occurred. However, restlessness was observed more frequently.

Conclusions Doxapram was applied to roughly a quarter of all very immature infants. Frequency and severity of apneas appeared to be reduced. Intubation because of apnea was avoided in a large proportion of infants. No severe side effects were recorded. More systematic studies on efficacy and safety of doxapram in premature infants are needed.

\section{RATIONAL USE OF ANTIBIOTICS IN NEWBORN}

doi:10.1136/archdischild-2012-302724.1650

0 Stanojlović, N Todorović, N Jovanović, N Miljković. Neonatology, Clinical Medical Center 'Zvezdara', Belgrade, Serbia

Side efects of antibiotics must always be considered, especialy in neonatal period. Our aim was to analise the antibiotic use in our neonatal ward(2nd level perinatal care).We analised number of babies who received therapy, indications and time of starting antibiotics, duration and antibiotis used, laboratory analysis (CBC, CRP, swabs and cultures of babies and mothers)) and discharge diagnosis in one-year period.

2299 babies were born in 2011, and $125(5.4 \%)$ recived antibiotics. $49 \%$ had risk factors for infection. In $62 \%$ therapy started in 1 st or 2 nd day of life, and average duration was 5-7 days, in $83 \%$. Ampicillin+Gentamycin was given in $82 \%$; Ampicillin for GBS colonisation and cefalosporines for UTI. There were no multiresistant strains.

\section{Diagnosis}

Respiratory: 43 (34.4\%),

Asphyxia: 17 (13.6\%),

Urinary tract infections: 15 (12\%),

Sepsis: 6 (4.8\%),

Others: 13 (10.4\%),

Without diagnosis: 31 (24.8\%).

Risk factors for infection were present in 20\% (ITU group) to $58 \%$ (group without diagnosis). Positive laboratory analysis were present from $42 \%$ (respiratory problems) to 100\% in sepsis and UTI.

Most of the children received therapy for clinical symptoms of infection, mostly $\mathrm{RD}$. The only single risk factor for starting the therapy was chorioamnionitis. Among children without diagnosis, 4 received short-course therapy based on risk factors, 6 because of GBS colonization, some had risk factors accompanied with positive laboratory findings and 10 babies because of positive laboratory findings only.

We noticed the decresement in antibiotis use in our hospital in past few years, specialy in profilactic use and thepary based on laboratory analysis. We consider clinical findings the most important criteria. But we can make further reduction by establishing firm criterias for antibiotic use, improving laboratory technics and probably shortening the duration of therapy in some children.

\section{ANGIOTENSIN II RECEPTOR ANTAGONIST RELATED FETOPATHY - A CASE REPORT}

doi:10.1136/archdischild-2012-302724.1651

K Wegleiter, E Griesmaier, M Waltner-Romen, R Trawoeger, U Kiechl-Kohlendorfer. Department of Paediatrics II, Medical University Innsbruck, Innsbruck, Austria

Fetal angiotensin II receptor antagonist exposure during pregnancy is associated with major congenital malformations including premature birth, oligohydramnios, acute renal failure, pulmonary hypoplasia and hypocalvaria. Fetopahty is mainly caused by renal insufficiency due to severe hypotension and disturbance of renal development. Therefore administration during the second and third trimester of pregnancy is contraindicated.
We report on a 35 year-old woman with arterial hypertension who was referred to our obstetrical department because of oligohydramnios. She reported to receive treatment with Olmesartanmedoxomil (5mg/day). The condition resolved after changing anti-hypertensive treatment to metoprolol at $266 / 7$ weeks of pregnancy. The patient was born at term by $\mathrm{C}$-section and showed the following signs of fetopathy: hyperechogenic multicystic kidneys and hypocalvaria. Renal function was normal, nevertheless arterial hypertension was present but treatment was not required. Discharge from hospital was possible at the age of 9 days. Regularly follow-up visits are necessary to monitor renal function and to evaluate long term effects.

Incidence of sartan-related fetopathy is unknown, therefore consistent reporting is mandatory. We present a case with mild presentation of symptoms, probably related to low therapeutic dosage and early change of antihypertensive treatment.

\section{EPIDEMIOLOGICAL STUDY ON ACUTE INTOXICATIONS IN THE ADMITTED CHILDREN}

doi:10.1136/archdischild-2012-302724.1652

${ }^{1} \mathrm{CE}$ Singer, ${ }^{1} \mathrm{P}$ Stancu, ${ }^{1} \mathrm{SC}$ Cosoveanu, ${ }^{1} \mathrm{~A}$ Botu, ${ }^{2} \mathrm{C}$ Cristea, ${ }^{2} \mathrm{~S}$ Costache. ${ }^{2} 2 \mathrm{nd}$ Pediatric Clinic, University of Medicine and Pharmacy Craiova; ${ }^{22} 2$ d Pediatric Clinic, Emergency County Hospital Craiova, Craiova, Romania

Objectives Study on the incidence of acute intoxications (AI) within general pathology and various parameters (sex, social environment, age, etiologic spectrum).

Material and Method A retrospective study of AI in the children aged $0-16$ years, admitted to the $2^{\text {nd }}$ Pediatric Clinic of the Emergency County Hospital Craiova from January $1^{\text {st }}$ to December $31^{\text {st }}$ 2011.

Results 95 children with various AI were admitted, representing $4.1 \%$ of the total admitted children; 41 (43.2\%) presented acute involuntary intoxications (AII), while 54 (56.8\%) acute voluntary intoxications (AVI).

AII: distribution of children according to sex $\mathrm{M} / \mathrm{F}=27 / 14$, social environment $\mathrm{U} / \mathrm{R}=18 / 23$, age group (years): $0-1 / 1-3 / 3-5 /$ 5-10/10-14/14-16=11/10/9/5/4/2; etiologic spectrum: drugs in 9 children, nitrates in 7 , carbon monoxide 7 , mushrooms 6 , corrosive substances 5 , insecticides/anti-parasitary 3 , ethylic alcohol 2 , medicinal alcohol 1 , and acetone 1 .

In AVI, the sex ratio was $M / F=21 / 33$, social environment $U /$ $\mathrm{R}=27 / 27$, age group (years): $5-10 / 10-14 / 14-16=12 / 17 / 25$. Causes of AVI: drugs in 32, ethylic alcohol 12, ethno-botanical 4, corrosive substances 3 , unknown causes 2 , caffeine 1 case. There were registered 2 deaths because of nitrates intoxication, in rural infants, aged 1 and 2 months.

Average period of hospitalization (days): in AII 4.79 \pm 3.12 (1-16), in AVI 3.25 $\pm 1.3(1-10)$

Conclusions AI represented $4.1 \%$ of the total admitted cases. Drugs represented the most frequent cause both in AII and AVI. AII were more frequent in males and rural areas; AVI prevailed in females. Deaths because of AI represented $2.1 \%$ of the total number of AI cases.

\section{ACUTE INTOXICATIONS WITH DRUG SUBSTANCES IN CHILDREN - A CLINICAL EPIDEMIOLOGICAL STUDY}

doi:10.1136/archdischild-2012-302724.1653

${ }^{1} \mathrm{CE}$ Singer, ${ }^{1} \mathrm{P}$ Stancu, ${ }^{1} \mathrm{SC}$ Cosoveanu, ${ }^{1} \mathrm{~A}$ Botu, ${ }^{2} \mathrm{~S}$ Costache, ${ }^{2} \mathrm{C}$ Cristea. ${ }^{2} 2 \mathrm{nd}$ Pediatric Clinic, University of Medicine and Pharmacy Craiova; ${ }^{2} 2$ nd Pediatric Clinic, Emergency County Hospital Craiova, Craiova, Romania

Objectives Incidence of acute drug intoxications (ADI) within general pathology and various parameters (sex, social environment, age group, etiologic spectrum, clinical form, average period of hospitalization). 